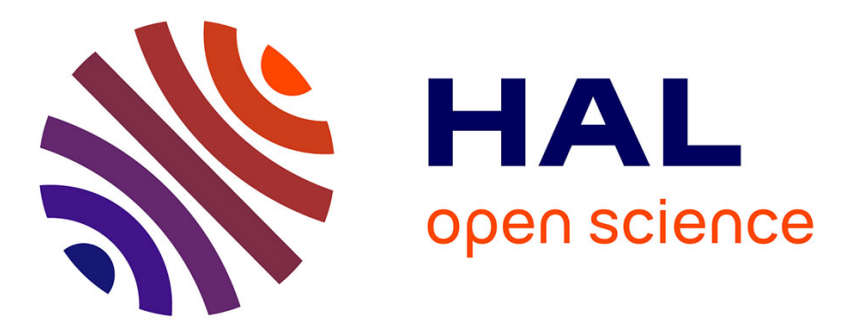

\title{
Syndrome néphrotique d'allure idiopathique après l'âge de 10 ans : intérêt de la biopsie rénale systématique au diagnostic
}

Anna Smith

\section{- To cite this version:}

Anna Smith. Syndrome néphrotique d'allure idiopathique après l'âge de 10 ans : intérêt de la biopsie rénale systématique au diagnostic. Sciences du Vivant [q-bio]. 2021. dumas-03261113

\section{HAL Id: dumas-03261113 https://dumas.ccsd.cnrs.fr/dumas-03261113}

Submitted on 15 Jun 2021

HAL is a multi-disciplinary open access archive for the deposit and dissemination of scientific research documents, whether they are published or not. The documents may come from teaching and research institutions in France or abroad, or from public or private research centers.
L'archive ouverte pluridisciplinaire HAL, est destinée au dépôt et à la diffusion de documents scientifiques de niveau recherche, publiés ou non, émanant des établissements d'enseignement et de recherche français ou étrangers, des laboratoires publics ou privés.

\section{(1) (1) $\$$}

Distributed under a Creative Commons Attribution - NonCommercial - NoDerivatives| 4.0 


\section{THESE DE DOCTORAT EN MEDECINE}

\section{DIPLOME D’ETAT}

Année 2021

Thèse présentée par Madame SMITH Anna, née le 19/07/1992 à Mantes-la-Jolie

Thèse soutenue publiquement le 01/06/2021

Syndrome néphrotique d'allure idiopathique après l'âge de 10 ans : Intérêt de la biopsie rénale systématique au diagnostic

Président

Membres du jury
M. le Professeur DE PARSCAU DU PLESSIX Loïc

Mme le Docteur DOSSIER Claire (directrice de thèse)

Mme le Docteur JAY Nadine

Mme le Docteur ROPARS Juliette

M. le Professeur LE MEUR Yannick 


\section{UNIVERSITE DE BRETAGNE OCCIDENTALE \\ FACULTE DE MEDECINE ET DES SCIENCES DE LA SANTE DE BREST}

Février 2021

Doyens honoraires

FLOCH Hervé $(\dagger)$

LE MENN Gabriel ( $\dagger$ )

SENECAIL Bernard

BOLES Jean-Michel

BIZAIS Yves ( $t$ )

DE BRAEKELEER Marc $(\dagger)$

Doyen

BERTHOU Christian

Professeurs émérites

BOLES Jean-Michel

Réanimation

BOTBOL Michel

Pédopsychiatrie

CENAC Arnaud

Médecine interne

COLLET Michel

Gynécologie obstétrique

JOUQUAN Jean

Médecine interne

LEFEVRE Christian

Anatomie

LEHN Pierre

Biologie cellulaire

MOTTIER Dominique

Thérapeutique

OZIER Yves

Anesthésiologie-réanimation

YOUINOU Pierre

Immunologie

Professeurs des Universités - Praticiens Hospitaliers de Classe Exceptionnelle

BERTHOU Christian

BRESSOLLETTE LUC

COCHENER-LAMARD Béatrice

DEWITTE Jean-Dominique

DUBRANA Frédéric

FEREC Claude

FOURNIER Georges

GENTRIC Armelle

GILARD Martine

GOUNY Pierre

KERLAN Véronique

LE MEUR Yannick

LE NEN Dominique

LEROYER Christophe

MANSOURATI Jacques

MERVIEL Philippe

MISERY Laurent

NONENT Michel

REMY-NERIS Olivier
Hématologie

Médecine vasculaire

Ophtalmologie

Médecine et santé au travail

Chirurgie orthopédique et traumatologique

Génétique

Urologie

Gériatrie et biologie du vieillissement

Cardiologie

Chirurgie vasculaire

Endocrinologie, diabète et maladies métaboliques

Néphrologie

Chirurgie orthopédique et traumatologique

Pneumologie

Cardiologie

Gynécologie obstétrique

Dermato-vénérologie

Radiologie et imagerie médicale

Médecine physique et réadaptation 


$\begin{array}{ll}\text { ROBASZKIEWICZ Michel } & \text { Gastroentérologie } \\ \text { SALAUN Pierre-Yves } & \text { Biophysique et médecine nucléaire } \\ \text { SARAUX Alain } & \text { Rhumatologie } \\ \text { TIMSIT Serge } & \text { Neurologie } \\ \text { WALTER Michel } & \text { Psychiatrie d'adultes }\end{array}$

Professeurs des Universités - Praticiens Hospitaliers de $1^{\text {ère }}$ Classe

AUBRON Cécile
BAIL Jean-Pierre
BEN SALEM Douraied
BERNARD-MARCORELLES Pascale
BEZON Éric
BLONDEL Marc
CARRE Jean-LuC
COUTURAUD Francis
DE PARSCAU DU PLESSIX Loïc
DELARUE Jacques
DEVAUCHELLE-PENSEC Valérie
DUEYMES Maryvonne
GIROUX-METGES Marie-Agnès
HU Weiguo
HUET Olivier
L'HER ErWan
LACUT Karine
MARIANOWSI Rémi
MONTIER Tristan
NOUSBAUM Jean-Baptiste
NEVEZ Gilles
PAYAN Christopher
PRADIER Olivier
SEIZEUR Romuald
STINDEL Éric

VALERI Antoine
Réanimation

Chirurgie digestive

Radiologie et imagerie médicale

Anatomie et cytologie pathologiques

Chirurgie thoracique et cardiovasculaire

Biologie cellulaire

Biochimie et biologie moléculaire

Pneumologie

Pédiatrie

Nutrition

Rhumatologie

Immunologie

Physiologie

Chirurgie plastique, reconstructrice et esthétique

Anesthésiologie-réanimation

Réanimation

Thérapeutique

Oto-rhino-laryngologie

Biologie cellulaire

Gastroentérologie

Parasitologie et mycologie

Bactériologie-virologie

Cancérologie

Anatomie

Biostatistiques, informatique médicale et technologies de communication

Urologie

Professeurs des Universités - Praticiens Hospitaliers de $2^{\text {ème }}$ Classe

\author{
ABGRAL Ronan \\ ANSART Séverine \\ BROCHARD Sylvain \\ BRONSARD Guillaume \\ CORNEC Divi \\ CORNEC-LE GALL Emilie \\ GENTRIC Jean-Christophe \\ HERY-ARNAUD Geneviève \\ IANOTTO Jean-Christophe \\ LE GAC Gérald \\ LE MARECHAL Cédric \\ LE ROUX Pierre-Yves \\ LE VEN Florent \\ LIPPERT Éric \\ THEREAUX Jérémie \\ TROADEC Marie-Bérengère
}

Biophysique et médecine nucléaire

Maladies infectieuses

Médecine physique et réadaptation

Pédopsychiatrie

Rhumatologie

Néphrologie

Radiologie et imagerie médicale

Bactériologie-virologie

Hématologie

Génétique

Génétique

Biophysique et médecine nucléaire

Cardiologie

Hématologie

Chirurgie digestive

Génétique 


$\begin{array}{ll}\text { JAMIN Christophe } & \text { Immunologie } \\ \text { MOREL Frédéric } & \text { Biologie et médecine du développement et de la } \\ \text { reproduction }\end{array}$

Maîtres de Conférences des Universités - Praticiens Hospitaliers de $1^{\text {ère }}$ Classe

$\begin{array}{ll}\text { BERROUIGUET Sofian } & \text { Psychiatrie d'adultes } \\ \text { BRENAUT Emilie } & \text { Dermato-vénéréologie } \\ \text { DE VRIES Philine } & \text { Chirurgie infantile } \\ \text { DOUET-GUILBERT Nathalie } & \text { Génétique } \\ \text { GUILLOU Morgane } & \text { Addictologie } \\ \text { HILLION Sophie } & \text { Immunologie } \\ \text { LE BERRE Rozenn } & \text { Maladies infectieuses } \\ \text { LE GAL Solène } & \text { Parasitologie et mycologie } \\ \text { LODDE Brice } & \text { Médecine et santé au travail } \\ \text { MAGRO Elsa } & \text { Neurochirurgie } \\ \text { MIALON Philippe } & \text { Physiologie } \\ \text { PERRIN Aurore } & \text { Biologie et médecine du développement et de la } \\ & \text { reproduction } \\ \text { PLEE-GAUTIER Emmanuelle } & \text { Biochimie et biologie moléculaire } \\ \text { QUERELLOU Solène } & \text { Biophysique et médecine nucléaire } \\ \text { ROBIN Philippe } & \text { Biophysique et médecine nucléaire } \\ \text { SCHICK Ulrike } & \text { Cancérologie } \\ \text { TALAGAS Matthieu } & \text { Histologie, embryologie et cytogénétique } \\ \text { UGUEN Arnaud } & \text { Anatomie et cytologie pathologiques } \\ \text { VALLET Sophie } & \text { Bactériologie-virologie } \\ \end{array}$

Maîtres de Conférences des Universités - Praticiens Hospitaliers de $2^{\text {ème }}$ Classe

$\begin{array}{ll}\text { ROUE Jean-Michel } & \text { Pédiatrie } \\ \text { SALIOU Philippe } & \text { Epidémiologie, économie de la santé et prévention } \\ \text { TROMEUR Cécile } & \text { Pneumologie }\end{array}$

Professeurs des Universités de Médecine Générale

LE FLOC'H Bernard
LE RESTE Jean-Yves

Maîtres de Conférences de Médecine Générale

BARAIS Marie

NABBE Patrice

Praticiens Hospitaliers Universitaires

BEAURUELLE Clémence
BAGACEAN Cristina
KERFANT Nathalie
ROPARS Juliette
THUILLIER Philippe

BEAURUELLE Clémence

BAGACEAN Cristin

THUILLIER Philippe
Bactériologie virologie

Hématologie

Chirurgie plastique

Pédiatrie

Endocrinologie, diabète et maladies métaboliques 
METGES Jean-Philippe Cancérologie

Maître de Conférences des Universités Associé

GURIEC Nathalie Nutrition

Professeurs des Universités Associés de Médecine Générale

BARRAINE Pierre

CHIRON Benoît

Maîtres de Conférences Associés de Médecine Générale

BEURTON-COURAUD LUCAS

DERRIENNIC JérémY

VIALA Jeanlin

Professeur des Universités

BORDRON Anne

Biologie cellulaire

Maîtres de Conférences des Universités

$\begin{array}{ll}\text { BERNARD Delphine } & \text { Biochimie et biologie moléculaire } \\ \text { BOUSSE Alexandre } & \text { Génie informatique, automatique et traitement du signal } \\ \text { DANY Antoine } & \text { Epidémiologie et santé publique } \\ \text { LE CORNEC Anne-Hélène } & \text { Psychologie } \\ \text { LANCIEN Frédéric } & \text { Physiologie } \\ \text { LE CORRE Rozenn } & \text { Biologie cellulaire } \\ \text { MIGNEN Olivier } & \text { Physiologie } \\ \text { MORIN Vincent } & \text { Electronique et informatique }\end{array}$

Maîtres de Conférences des Universités Contractuels LRU

GHIS MALFILATRE Marie Sociologie démographie

MERCADIE Lolita Psychologie

Attachée Temporaire d'Enseignement et de Recherche

GHANEM Rosy Biochimie et biologie moléculaire

Professeurs Certifiés / Agrégés du second degré
MONOT Alain
Français
RIOU Morgan
Anglais

Professeurs Agrégés du Val-de-Grâce (Ministère des Armées)

DULOU Renaud Neurochirurgie


Maîtres de Stages Universitaires - Référents (Ministère des Armées)

LE COAT Anne

SCELLOS Olivia
Médecine Générale / Urgence

Médecine Générale 
Table des matières

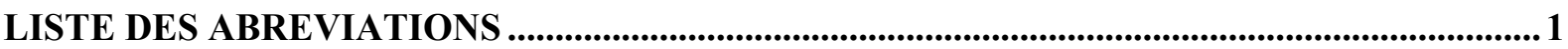

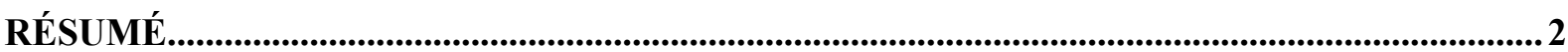

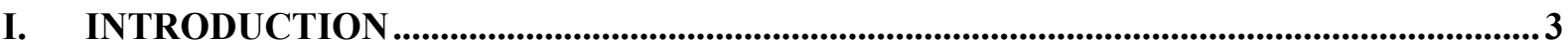

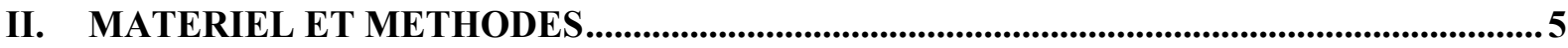

A. POPULATION DE L'ETUDE ET CRITERES D'INCLUSION/EXCLUSION............................................

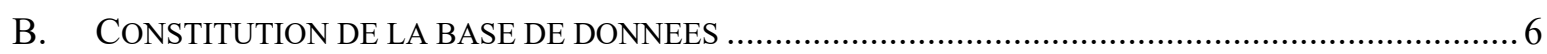

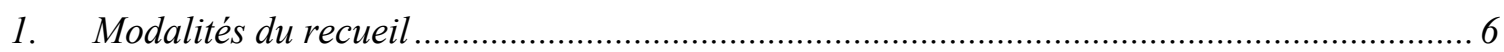

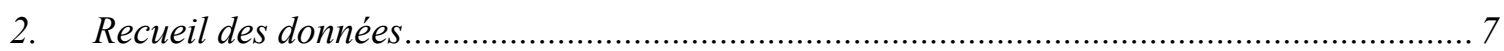

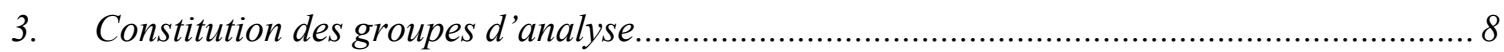

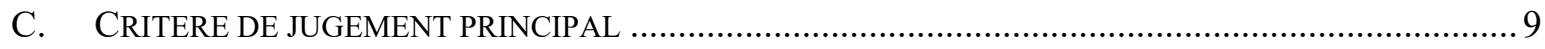

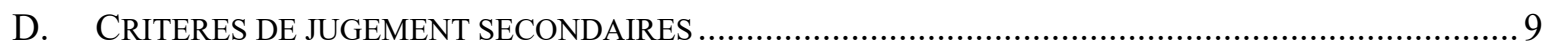

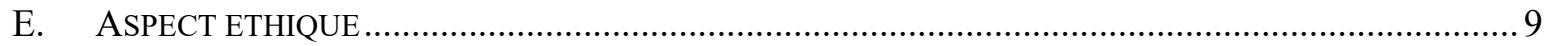

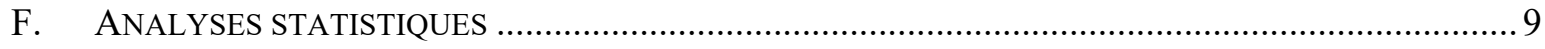

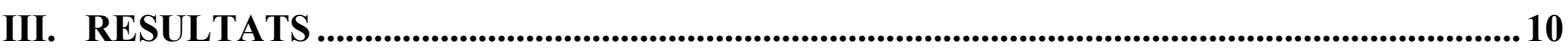

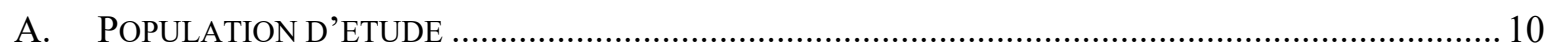

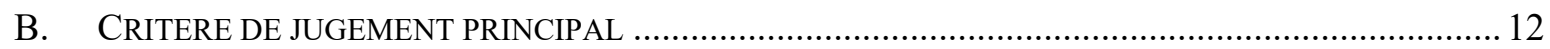

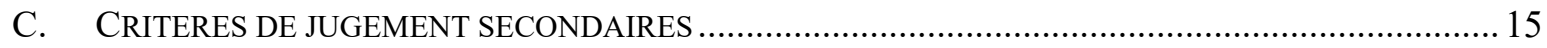

1. Répartition de l'âge et des origines ethniques en fonction du diagnostic ............................ 15

2. Description des complications des biopsies ................................................................... 18

3. Recherche d'un éventuel impact d'une PBR différée ........................................................ 18

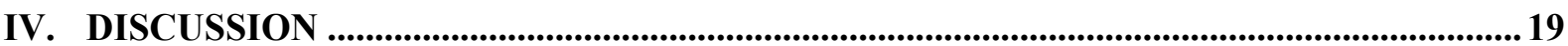

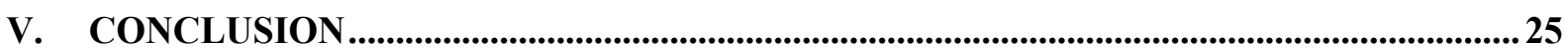

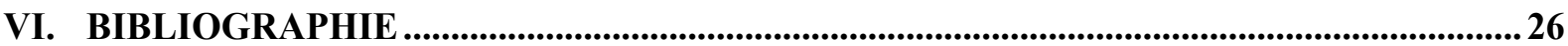




\section{Liste des abréviations}

SN : Syndrome néphrotique

SNI : Syndrome néphrotique idiopathique

ISKDC : The International Study of Kidney Disease in Children

APN : Arbeitsgemeinschaft für Padiatrische Nephrologie

PBR : Ponction biopsie rénale

FS : Forme secondaire du syndrome néphrotique

GEM : Glomérulopathie extra-membraneuse

FAN : Facteur anti-nucléaire

ANCA : Anticorps anticytoplasme des polynucléaires neutrophiles

HTA : Hypertension artérielle

IR : Insuffisance rénale

LGM : Lésions glomérulaires minimes

HSF : Hyalinose segmentaire et focale

PMD : Prolifération mésangiale diffuse 


\title{
Idiopathic nephrotic syndrome after 10 years-old: Is systematic renal biopsy appropriate?
}

\author{
Anna Smith ${ }^{1}$, Eric Xu ${ }^{2}$, Olivia Boyer ${ }^{3}$, Jean Daniel Delbet ${ }^{4}$, Michel Peuchmaur ${ }^{5}$, Julien Hogan ${ }^{1}$, Claire \\ Dossier $^{1}$ \\ 1- Pediatric Nephrology, Robert-Debre hospital, APHP, Paris, France \\ 2- Augustin Morvan Hospital, Brest, France \\ 3- Pediatric Nephrology, Necker Hospital, APHP, Paris, France \\ 4- Pediatric Nephrology, Armand-Trousseau Hospital, APHP, Paris, France \\ 5- Anatomopathology, Robert-Debre Hospital, APHP, Paris, France
}

\section{Introduction:}

Renal biopsy (RB) is recommended at diagnosis for children presenting with isolated nephrotic syndrome (NS) after the age of 10 - 12 years old, unlike in younger children, because of a higher frequency of other diagnosis than MCD or FSGS. Recent reports, local practices and new serological biomarkers for membranous nephropathy now question this approach. Therefore, the objective of our study was to evaluate causes of NS and indications for RB in a population-based cohort of adolescents with NS.

\section{Material and methods:}

We conducted a retrospective multicenter study, including children aged 10 to 18 years diagnosed with idiopathic NS between December 2007 and June 2020 in 35 pediatric and 3 pediatric nephrology (PN) departments of the Paris area (NEPHROVIR network) and patients with idiopathic-looking NS biopsied in the 3 PN departments.

\section{Results:}

Hundred and three children were included (54 males). Mean age at diagnosis was 13,4 \pm 2 years. A renal biopsy was performed in 76 patients: at presentation because of atypical symptoms (such as hypertension, macroscopic hematuria, organic renal failure) in 15 or for no other reason than age in 39, and after 4 weeks for steroid resistance $+/$ atypies in 22 patients. Histological findings were MCD ( $n=49)$, FSGS ( $n=13)$, mesengial proliferation $(n=3)$, membranous nephropathy $(n=6)$ and $\operatorname{IgAN}(n=5)$.

\section{Conclusions:}

NS was secondary in only $11 \%$ of adolescents presenting with idiopathic-like NS, and first line treatment with oral prednisone was appropriate in all cases. In addition, more than $40 \%$ of RB could have been avoided in steroid sensitive NS patients. Finally, we propose that RB should only be performed in case of atypical clinical or biological history or steroid resistance after 4 weeks, as in the younger population.

RB: Renal biopsy, NS : Nephrotic syndrome, MCD : Minimal-change disease (LGM), FSGS : Focal segmental glomerulosclerosis (HSF), PN : Pediatric nephrology, IgAN : IgA nephropathy 


\section{Introduction}

Le syndrome néphrotique ( $\mathrm{SN}$ ) est une néphropathie glomérulaire de cause primaire (idiopathique) ou de cause secondaire (Table 1). La fréquence de ses étiologies est variable selon l'âge du patient, orientant ainsi la prise en charge au diagnostic.

Chez les enfants de moins d'un an, l'origine génétique représente $60 \%$ des syndromes néphrotiques diagnostiqués. Plus rarement, des causes infectieuses (toxoplasmose, syphilis, CMV) peuvent être rapportées. ${ }^{1-3}$

Chez l'enfant entre 1 et 10 ans, la forme idiopathique est la cause de plus de $90 \%$ des SN et est sensible au traitement par corticoïdes dans plus de $90 \%$ des cas. L'approche diagnostique et thérapeutique du syndrome néphrotique idiopathique (SNI) chez l'enfant entre 1 et 10 ans résulte d'une série d'études depuis les années 60-70 avec la collaboration de l'étude des maladies rénales chez l'enfant (ISKDC) et l'Arbeitgemeinschaft für padiatrische nephrologie (APN) permettant d'affirmer qu'il n'y a pas de corrélation entre les présentations histologiques et le pronostic rénal. En revanche, la réponse au traitement par corticoïdes est prédictive de l'évolution. Par conséquent, la réponse aux corticoïdes constitue un test diagnostique évitant la réalisation d'une biopsie rénale inutile dans la majorité des SNI chez l'enfant de 1 à 10 ans..$^{4-7}$ La ponction biopsie rénale (PBR) sera réalisée devant une suspicion d'un syndrome néphrotique de forme secondaire (FS), d'une hématurie macroscopique, d'une insuffisance rénale organique ou en cas de corticorésistance. ${ }^{8}$

Chez l'adolescent et l'adulte, la fréquence des diagnostics différentiels du SNI serait plus élevée. En effet, les SN de forme secondaire représenteraient jusqu'à 50\% des SN chez l'adolescent, d'après une ancienne étude de $1978^{4}$, et jusqu'à $80 \%$ chez l'adulte ${ }^{9,10}$, ce pourquoi, la PBR est indiquée d'emblée au diagnostic. ${ }^{8,11}$

Au cours de ces dernières années, cette démarche diagnostique a été questionnée ${ }^{12}$ mais reste la conduite à tenir recommandée.

Chez l'adulte, la glomérulonéphrite extra-membraneuse primitive (GEM), principal diagnostic différentiel, a bénéficié ces dernières années d'avancées diagnostiques avec l'identification des anticorps anti-PLA2R détectés dans $70-80 \%$ des cas sur un simple bilan sanguin. ${ }^{13-15} \mathrm{Ceci}$ a conduit à remettre en question la stratégie diagnostique du SN de l'adulte et de ne discuter la réalisation de la PBR qu'en cas de sérologie PLA2R négative. ${ }^{14,15}$

Les données de la cohorte francilienne NEPHROVIR-1 entre 2007 et 2010, ont montré que seulement $50 \%$ des enfants de plus de 10 ans avaient bénéficié d'une biopsie rénale. Une des explications avancées est que la rémission était obtenue sous corticoïdes avant que le geste de biopsie rénale n'ait pu être organisé, confirmant ainsi une forme idiopathique sur un test diagnostique comme chez l'enfant plus jeune. 
De surcroit, la PBR est un geste invasif et douloureux qui nécessite une hospitalisation dans un centre spécialisé et peut entrainer des complications, en particulier hémorragiques. ${ }^{16}$

$\mathrm{Au}$ total, ces différents éléments font discuter la réalisation systématique d'une biopsie rénale sur le seul critère d'âge chez l'adolescent et l'enfant de plus de 10 ans.

Notre hypothèse est que les biopsies rénales réalisées sur le seul critère d'âge ne retrouveraient que des histologies en lien avec un SNI et seraient donc dispensables. L'objectif principal de notre travail était d'étudier l'intérêt de la biopsie rénale systématique dans la stratégie diagnostique chez les enfants de plus de 10 ans se présentant avec un SN.

Table 1. Principales causes de syndrome néphrotique secondaire

\begin{tabular}{ll}
\hline Génétique & Syndromique ou non syndromique \\
Infections & Toxoplasmose, syphilis, VIH, VHB, VHC... \\
Maladies auto-immunes & Lupus, vascularite à ANCA, purpura rhumatoide... \\
Maladies systémiques & Diabète... \\
Maladies rénales & $\begin{array}{l}\text { Glomérulopathie extra-membraneuse, néphropathie à IgA, } \\
\text { glomérulopathie membrano-proliférative... }\end{array}$ \\
Maladies tumorales & Lymphome, leucémie... \\
Traitements/toxiques & $\begin{array}{l}\text { Anti-inflammatoires non stéroïdiens, bisphosphonates, pénicilline, } \\
\text { intoxication aux sels d'or/mercure, lithium... }\end{array}$ \\
\hline
\end{tabular}




\section{Matériel et méthodes}

\section{A. Population de l'étude et critères d'inclusion/exclusion}

Nous avons conduit une étude rétrospective de type cohorte observationnelle, menée de décembre 2007 à juin 2020, impliquant les 39 centres de recrutement de la cohorte NEPHROVIR ${ }^{17}$ (Figure 1), dont les 3 centres de référence de néphrologie pédiatrique d'Île-De-France (Hôpital RobertDebré, Hôpital Necker-Enfants malades, Hôpital Armand-Trousseau).

Figure 1. Carte d'Île-De-France représentant les sites de recrutement de la cohorte NEPHROVIR

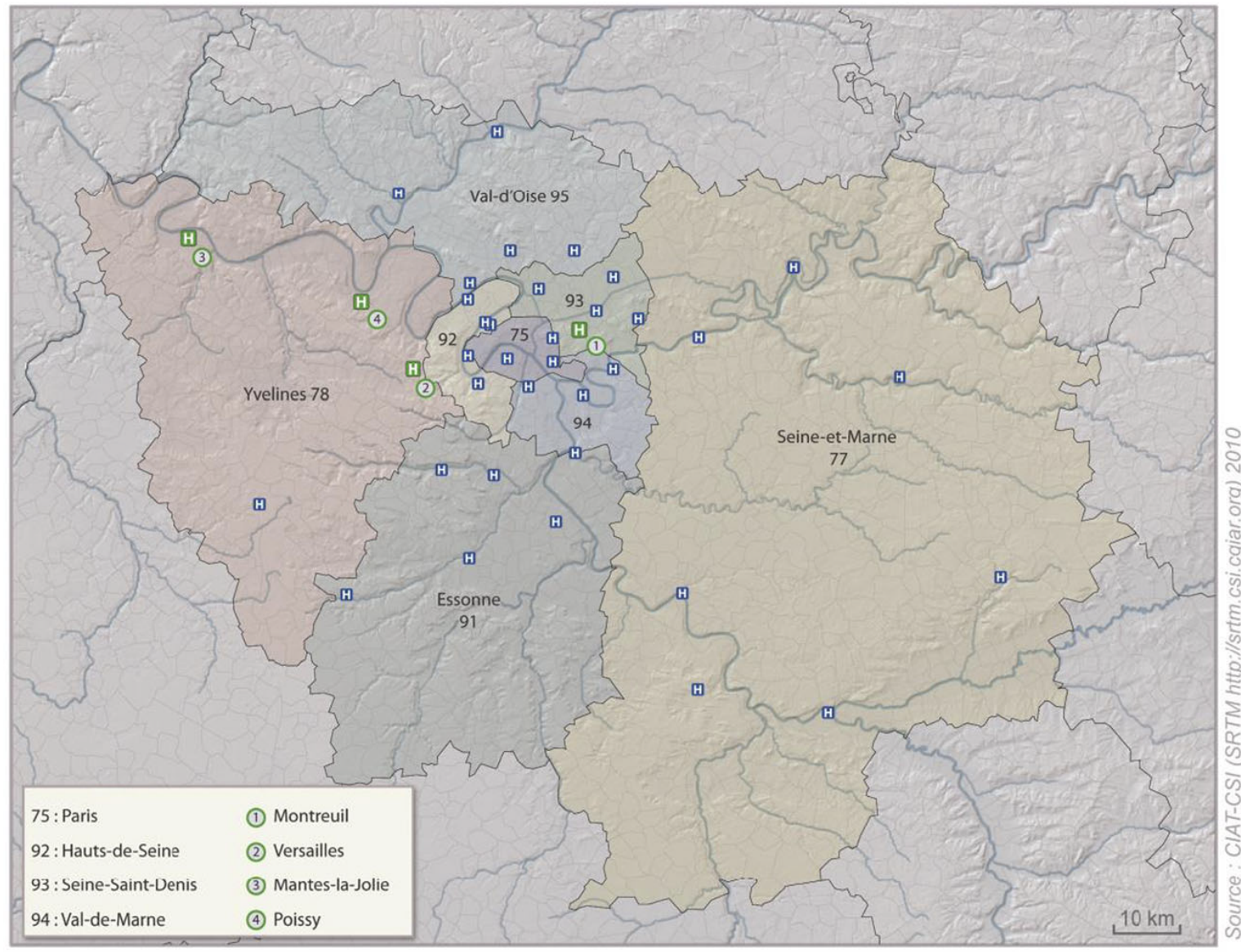


Les critères d'inclusion étaient :

- Les enfants âgés de 10 à 18 ans résidant en Île-De-France.

- Pris en charge et/ou suivis dans un des 3 services de néphrologie pédiatrique d'Île-De-France (centre hospitalier Robert-Debré, Armand-Trousseau, Necker-Enfants malades).

- Ayant présenté un syndrome néphrotique, défini par l'association d'une hypoalbuminémie < $30 \mathrm{~g} / \mathrm{L}$ et d'une protéinurie (caractérisée par un rapport protéinurie/créatininurie $>0.20 \mathrm{~g} / \mathrm{mmol}$ ou par une protéinurie $>50 \mathrm{mg} / \mathrm{kg} / \mathrm{j}$ ), sur reins natifs.

- Diagnostiqués entre décembre 2007 et juin 2020.

Les critères d'exclusion étaient :

- Les patients déjà diagnostiqués et suivis pour une maladie rénale ou systémique associée à un SN secondaire.

- Les patients ayant présenté des signes rénaux avant le diagnostic de SN (hématurie macroscopique récidivante, hématurie microscopique persistante, protéinurie chronique, insuffisance rénale chronique, hypertension artérielle).

- Les patients ayant présenté des signes cliniques extrarénaux (atteinte articulaire, cutanée, pulmonaire...) et/ou des signes biologiques (cytopénie, abaissement de la fraction C3 du complément, présence de facteur anti-nucléaire (FAN), présence d'anticorps anticytoplasme des polynucléaires neutrophiles (ANCA), sérologie VIH, VHB ou VHC positive) au diagnostic orientant vers une cause secondaire et dont la motivation de la réalisation d'une PBR était de déterminer l'étiologie du SN secondaire.

- Les patients ayant présenté une insuffisance rénale terminale au diagnostic.

\section{B. Constitution de la base de données}

\section{Modalités du recueil}

Nous avons recueilli les données des patients présentant un SN par l'intermédiaire de différentes sources d'identifications :

- Recherche des patients biopsiés sur reins natifs pour un SN dans les registres de biopsie rénale des 3 centres de néphrologie pédiatrique sur la période décembre 2007 à juin 2020.

- Recherche des patients présentant un SN dans la base de données de la cohorte NEPHROVIR$2^{17,18}$, par l'intermédiaire du logiciel DR WAREHOUSE (base de données informatisée de l'hôpital Necker-Enfants malades), ainsi que dans la base de données BAMARA (Banque nationale de données des maladies rares) des 3 centres. 


\section{Recueil des données}

Toutes les données cliniques concernant les patients ont été collectées de façon rétrospective. Nous avons recueilli les données suivantes :

- Données démographiques : Âge au diagnostic, sexe, ethnie.

- Données cliniques au diagnostic : La date du diagnostic (correspondant à la date de la première hospitalisation), la présentation clinique au diagnostic, la présence de signes néphrologiques comme : l'hypertension artérielle (HTA) ayant nécessité la mise en place d'un traitement antihypertenseur, la présence d'une hématurie microscopique (nombre de globules rouges dans les urines entre 10000 - 300 000/ml) ou macroscopique (nombre de globules rouges dans les urines > à 300 000/ml), la présence d'une insuffisance rénale (IR) fonctionnelle (se corrigeant après la correction du volume intravasculaire) ou organique (ne se corrigeant pas malgré la correction du volume intravasculaire ou nécessitant la mise en dialyse transitoire).

- Données histologiques : La date de réalisation de la PBR, les résultats histologiques, les complications de la biopsie (nombre, type, conséquence).

- Données d'évolution et sur la réponse initiale aux corticoïdes : La date du début d'un traitement par corticoïdes, une cortico-sensibilité (définie par la mise en rémission à la suite d'une corticothérapie de 4 semaines per os $+/$ - associée à une série de 3 bolus de corticoïdes intraveineux), une corticorésistance (définie par l'absence de rémission après 4 semaines de traitement par corticoïdes per os $+/$ - associée à 3 bolus de corticoïdes intraveineux), le bilan d'auto-immunité réalisé à la découverte d'un SN secondaire, la réalisation d'une recherche génétique en cas de corticorésistance et son résultat. 


\section{Constitution des groupes d'analyse}

Nous avons recherché dans l'ensemble des données de chaque patient, les indications ayant justifié la réalisation de la PBR, en accord avec le $\mathrm{PNDS}^{8}$ : que ce soit l'âge (plus de 10 ans) ou les autres critères tels qu'un tableau atypique ou une corticorésistance (Table 2).

Table 2. Indications de PBR autres que l'âge

\section{Tableau atypique}

- Histoire clinique atypique

- Présentation au diagnostic d'au moins un signe néphrologique suivant : HTA nécessitant un traitement antihypertenseur ; hématurie macroscopique ; insuffisance rénale organique

\section{Évolution vers une corticorésistance}

- Patients ayant eu une PBR au diagnostic et qui, par la suite, ont présenté une corticorésistance

- Patients ayant eu une PBR après avoir présenté une corticorésistance

- Patients suspects d'une corticorésistance (rechute précoce dans le premier mois de traitement par corticoïdes avec persistance d'une protéinurie à J28)

PBR : Ponction biopsie rénale, HTA : Hypertension artérielle.

Deux groupes d'analyse ont été constitués :

- Le groupe A correspond à l'ensemble des patients ayant présenté un ou plusieurs critères de réalisation d'une PBR autres que l'âge : la présence d'un tableau atypique et/ou d'une corticorésistance.

- Le groupe B est constitué de patients ayant eu une PBR seulement pour le critère d'âge, c'està-dire qu'ils n'ont ni présenté de tableau atypique ni d'évolution vers une corticorésistance. 


\section{Critère de jugement principal}

Le critère de jugement principal de l'étude était les résultats histologiques dans les différents groupes.

\section{Critères de jugement secondaires}

Les critères de jugement secondaires étaient :

- Décrire la répartition des FS et des SNI ainsi que les différentes lésions histologiques en fonction de l'âge au diagnostic et des origines ethniques.

- Décrire les complications de la PBR.

- Identifier les situations dans lesquelles un retard de réalisation de la PBR aurait pu avoir un impact sur le pronostic des patients présentant une FS.

\section{E. Aspect éthique}

Aucun sujet (ou leurs représentants légaux) ne s'est opposé à l'usage de leurs données médicales dans un but de recherche clinique. Le protocole d'étude est en cours de soumission à un comité d'éthique local indépendant.

\section{F. Analyses statistiques}

Les données recueillies ont été synthétisées dans un tableur Excel. Les données épidémiologiques de la cohorte ont été analysées selon les deux principaux groupes constitués (groupe A et groupe B) ainsi que le groupe des patients non biopsiés. Les données sont présentées en moyenne (+/- écart type), en médiane [min ; max], ou en nombre de patients. Des tests sur la distribution des données quantitatives ont été réalisés afin de pouvoir choisir les tests de comparaison appropriés (test paramétrique ou test non paramétrique). Toutes les analyses statistiques ont été réalisées grâce au logiciel JMP 14.0 (SAS). 


\section{Résultats}

\section{A. Population d'étude}

Au total, 110 patients ont été inclus dans notre étude. Pour 7 d'entre eux, les données concernant la PBR étaient manquantes. Sur les 103 patients analysés, l'âge moyen était de 13,4 ans et le ratio Garçon/Fille à 1,1. Au total, 76 patients ont eu une biopsie rénale (Figure 2).

Le groupe A était constitué de 43 patients qui présentaient au moins un critère de réalisation de la PBR autre que l'âge (tableau atypique et/ou corticorésistance).

Le groupe B était constitué de 33 patients qui ne présentaient ni tableau atypique, ni corticorésistance (PBR réalisée sur seul critère d'âge).

Figure 2. Diagramme de flux

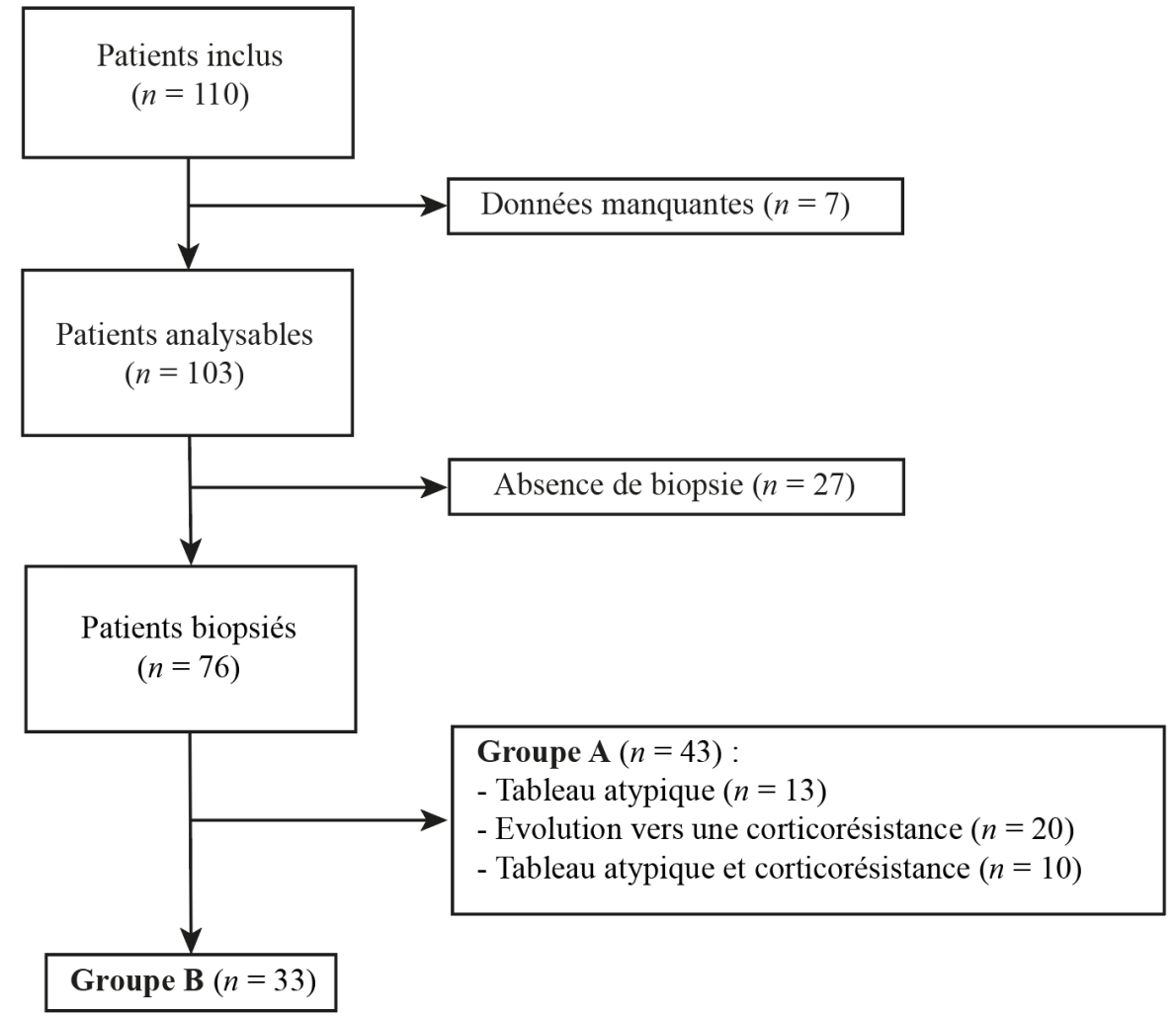

Les données concernant la population de l'étude sont présentées dans la Table 3. Il n'y avait pas de différence de sexe entre les différents groupes $(\mathrm{p}=1)$. Les patients du groupe A étaient plus jeunes que les patients du groupe $B(p=0,01)$. Les patients non biopsiés étaient significativement plus jeunes que les patients des groupes A et B (respectivement $p=0,0004$ et $\mathrm{p}<0,0001)$. 
Table 3. Description de la population d'étude

\begin{tabular}{|c|c|c|c|c|}
\hline & $\begin{array}{l}\text { Groupe A } \\
(\mathrm{N}=43)\end{array}$ & $\begin{array}{l}\text { Groupe B } \\
(\mathrm{N}=33)\end{array}$ & $\begin{array}{c}\text { Patients non } \\
\text { biopsiés } \\
(\mathrm{N}=27) \\
\end{array}$ & $\begin{array}{c}\text { Total } \\
(\mathrm{N}=103)\end{array}$ \\
\hline \multicolumn{5}{|l|}{ Données démographiques } \\
\hline Age au diagnostic (ans)* & $13,4 \pm 2,1$ & $14,5 \pm 1,5 * *$ & $11,5 \pm 1,4^{* * *}$ & $13,4 \pm 2$ \\
\hline Sexe masculin (n) & 23 & 18 & 13 & 54 \\
\hline \multicolumn{5}{|l|}{ Origine ethnique (n) } \\
\hline Caucasienne & 12 & 11 & 5 & 28 \\
\hline Afrique du Nord/Moyen orient & 5 & 6 & 8 & 19 \\
\hline Afrique sub-saharienne/Antilles & 17 & 7 & 4 & 28 \\
\hline Asie du Sud-est & 2 & 2 & 2 & 6 \\
\hline Asie de l'Ouest & 2 & 1 & 2 & 5 \\
\hline Autres & 1 & 0 & 0 & 1 \\
\hline NA & 4 & 6 & 6 & 16 \\
\hline \multicolumn{5}{|l|}{$\begin{array}{l}\text { Données cliniques au diagnostic } \\
\text { Histoire clinique (n) }\end{array}$} \\
\hline Typique & 36 & 33 & 27 & 96 \\
\hline Atypique & 7 & 0 & 0 & 7 \\
\hline HTA traitée au diagnostic (n) & 10 & 0 & 1 & 11 \\
\hline \multicolumn{5}{|l|}{ Hématurie (n) } \\
\hline Absence d'hématurie & 17 & 19 & 6 & 42 \\
\hline Microscopique & 12 & 13 & 4 & 29 \\
\hline Macroscopique & 11 & 0 & 2 & 13 \\
\hline \multicolumn{5}{|l|}{ IR initiale (n) } \\
\hline Absence d'IR initiale & 28 & 30 & 12 & 70 \\
\hline Fonctionnelle & 11 & 3 & 0 & 14 \\
\hline Organique & 2 & 0 & 0 & 2 \\
\hline \multicolumn{5}{|l|}{ Données histologiques } \\
\hline Biopsie rénale réalisée (n) & 43 & 33 & 0 & 76 \\
\hline Au diagnostic $(<\mathrm{J} 28)$ & 22 & 32 & 0 & 54 \\
\hline Différée (> J28) & 21 & 1 & 0 & 22 \\
\hline \multicolumn{5}{|l|}{$\begin{array}{l}\text { Suivi et réponse au traitement } \\
\text { Réponse aux corticoïdes (n) }\end{array}$} \\
\hline Corticosensible & 11 & 33 & 27 & 71 \\
\hline Corticorésistant & 27 & 0 & 0 & 27 \\
\hline $\begin{array}{l}\text { Pas de traitement } \\
\text { débuté/incomplet }\end{array}$ & 5 & 0 & 0 & 5 \\
\hline
\end{tabular}

* moyenne \pm écart-type

** $\mathrm{p}=0,01$ en comparaison avec la moyenne d'âge du groupe A

$* * * \mathrm{p}=0,0004$ et $\mathrm{p}<0,0001$ respectivement en comparaison à la moyenne d'âge du groupe $\mathrm{A}$ et $\mathrm{B}$

HTA : Hypertension artérielle nécessitant un traitement, IR : Insuffisance rénale,

NA : Données non disponibles. 
Concernant les patients du groupe A :

- Sept histoires atypiques ont été décrites dont une rémission spontanée suivie d'une rechute précoce dans le premier mois, 2 descriptions d'œdèmes chroniques (de plus de 6 mois) et 4 découvertes fortuites d'un SN lors d'une hospitalisation pour un autre motif : lors de la découverte d'un diabète, à la suite d'une crise de paludisme, à la suite d'une pyélonéphrite aigue, lors d'un bilan d'exploration pour énurésie primaire.

- Dix patients ont présenté une HTA nécessitant un traitement au diagnostic, 11 ont présenté une hématurie macroscopique et 2 ont présenté une IR organique.

- Sept patients ont été biopsiés au moment du diagnostic mais n'ont pas présenté de rémission après un mois de traitement par corticoïdes $+/$ - bolus. Treize patients ont été biopsiés après avoir présenté une corticorésistance, dont trois avec rémission initiale et qui ont présenté une rechute précoce sous corticoïdes dans le premier mois de traitement.

La biopsie rénale était réalisée après un délai médian de 11,5 jours $[0 ; 122]$ pour les patients du groupe A et de 4 jours $[0 ; 31]$ pour les patients du groupe B.

\section{B. Critère de jugement principal}

Sur l'ensemble de la cohorte, le diagnostic de SNI (patients biopsiés ou non) a représenté $88,4 \%$ et les FS 11,6\%. Concernant les patients biopsiés, les données histologiques en fonction des différents groupes sont présentées dans la Table 4.

Table 4. Données histologiques des patients biopsiés

\begin{tabular}{lccc}
\hline & $\begin{array}{c}\text { Groupe A } \\
(\mathrm{N}=43)\end{array}$ & $\begin{array}{c}\text { Groupe } \mathrm{B} \\
(\mathrm{N}=33)\end{array}$ & $\begin{array}{c}\text { Total } \\
(\mathrm{N}=76)\end{array}$ \\
\hline $\begin{array}{l}\text { Syndrome néphrotique idiopathique } \\
\text { LGM }\end{array}$ & $31(72,1)$ & $33(100)$ & $64(84,2)$ \\
HSF & $19(44,2)$ & $30(90,9)$ & $49(64,5 / 76,6)$ \\
PMD & $10(23,3)$ & $2(6,1)$ & $12(15,8 / 18,8)$ \\
& $2(4,7)$ & $1(3)$ & $3(3,9 / 4,7)$ \\
Formes secondaires & & & \\
$\quad$ GEM & $12(27,9)$ & $0(0)$ & $12(15,8)$ \\
$\quad$ Néphropathie à IgA & $6(14)$ & $0(0)$ & $6(7,9 / 50)$ \\
$\quad$ Syndrome néphrotique génétique* & $5(11,6)$ & $0(0)$ & $5(6,6 / 41,7)$ \\
\hline Les don & $1(2,3)$ & $0(0)$ & $1(1,3 / 8,3)$ \\
\hline
\end{tabular}

Les données sont présentées en $\mathrm{n}$ (\% Total / \% Sous-groupe)

LGM : Lésions glomérulaires minimes, HSF : Hyalinose segmentaire et focale,

PMD : Prolifération mésangiale diffuse, GEM : Glomérulonéphrite extra-membraneuse.

*Mutation génétique SMARCAL1 avec lésions d'HSF retrouvées à l'histologie. 
La biopsie réalisée pour critère d'âge seul (Groupe B) concernait 43,4\% de l'ensemble des patients biopsiés. Aucune FS n'a été retrouvée dans le groupe B.

Soixante-quatre patients biopsiés ont présenté des lésions histologiques d'un SNI. La lésion histologique la plus fréquente, soit 76,6\% des cas, était des lésions glomérulaires minimes (LGM) et des lésions de hyalinose segmentaire et focale (HSF) étaient retrouvées dans 18,8\% des cas.

Parmi les 12 patients ayant présenté une FS : 6 patients ont présenté des lésions histologiques d'une GEM, 5 ont présenté des lésions histologiques d'une néphropathie à $\operatorname{IgA}$ et le dernier patient a présenté des lésions d'HSF à l'histologie avec une mutation du gène SMARCALl (syndrome de Schimcke) retrouvée au bilan génétique permettant de conclure à un syndrome néphrotique génétique.

Dans cette cohorte, nous avons observé que 54 patients ont été biopsiés au diagnostic, 22 patients ont eu une biopsie différée et 27 patients, tous cortico-sensibles, n'ont jamais été biopsiés (Figure 3). 


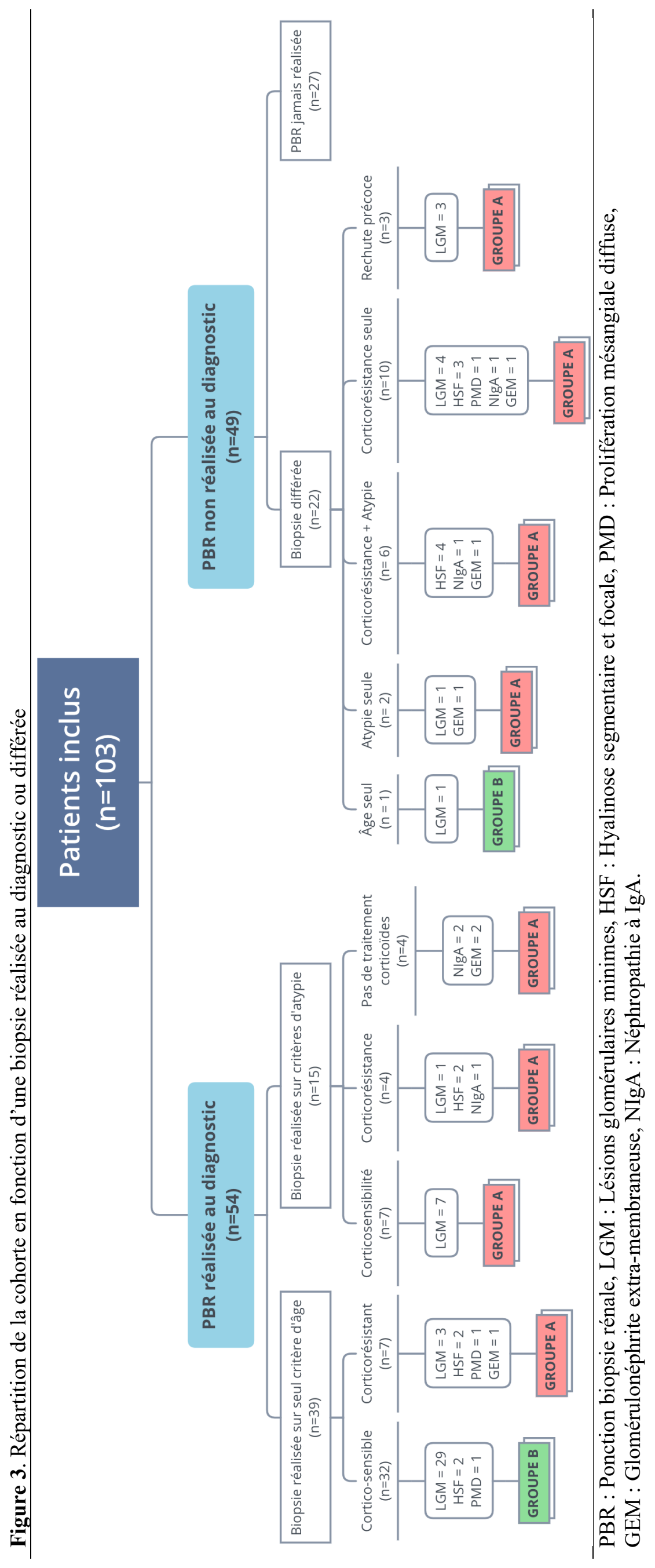


Tous les patients corticorésistants ont été biopsiés ( $\mathrm{n}=27)$ : 8 LGM, $11 \mathrm{HSF}, 2$ prolifération mésangiale diffuse (PMD), 3 néphropathies à IgA et 3 GEM. Parmi les 71 patients corticosensibles : 44 ont été biopsiés (41 LGM, 2 HSF, 1 PMD) et 27 n'ont pas eu de biopsie. Cinq patients (2 néphropathies à IgA et 3 GEM) n'ont pas pu être classés en fonction de leurs réponses aux corticoïdes (traitement non débuté ou incomplet).

Une hématurie microscopique a été retrouvée chez $29 \%$ des patients corticosensibles et $26 \%$ des patients corticorésistants. D'autre part, $27 \%$ des SNI ont présenté une hématurie microscopique au diagnostic et $33 \%$ des patients FS.

\section{Critères de jugement secondaires}

1. Répartition de l'âge et des origines ethniques en fonction du diagnostic

La moyenne d'âge des patients SNI était de 13,4 ans et des patients FS était de 13,7 ans $(\mathrm{p}=0,74)$. La répartition des FS et SNI selon l'âge (Figure 4) ne met pas en évidence de seuil d'âge permettant leur distinction. Concernant les répartitions histologiques selon l'âge, il ne semble pas exister d'augmentation des lésions d'HSF avec l'âge. 
Figure 4. Histogramme en fonction de l'âge

A. Répartition des SNI et des FS en fonction de l'âge

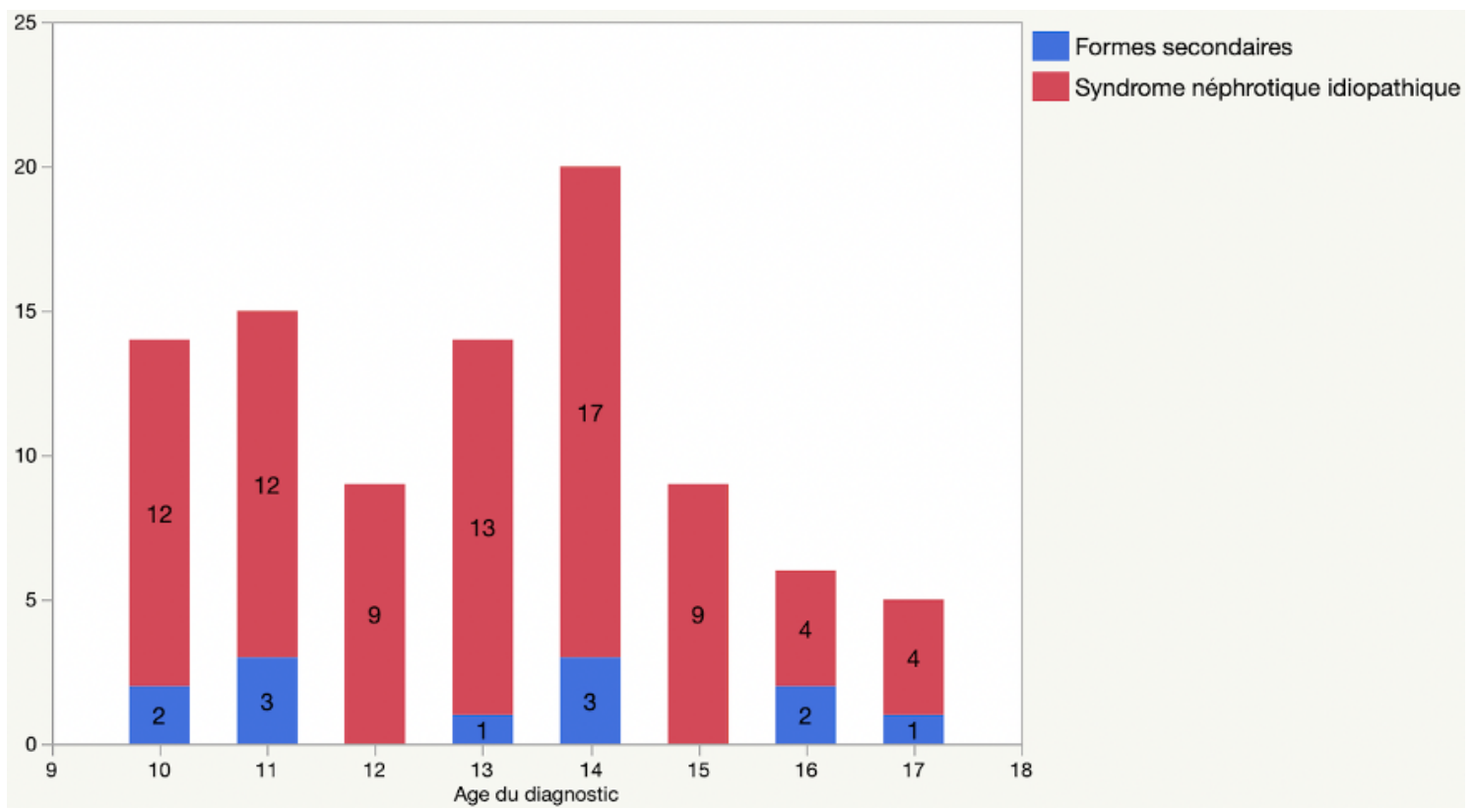

B. Répartition des lésions histologiques en fonction de l'âge

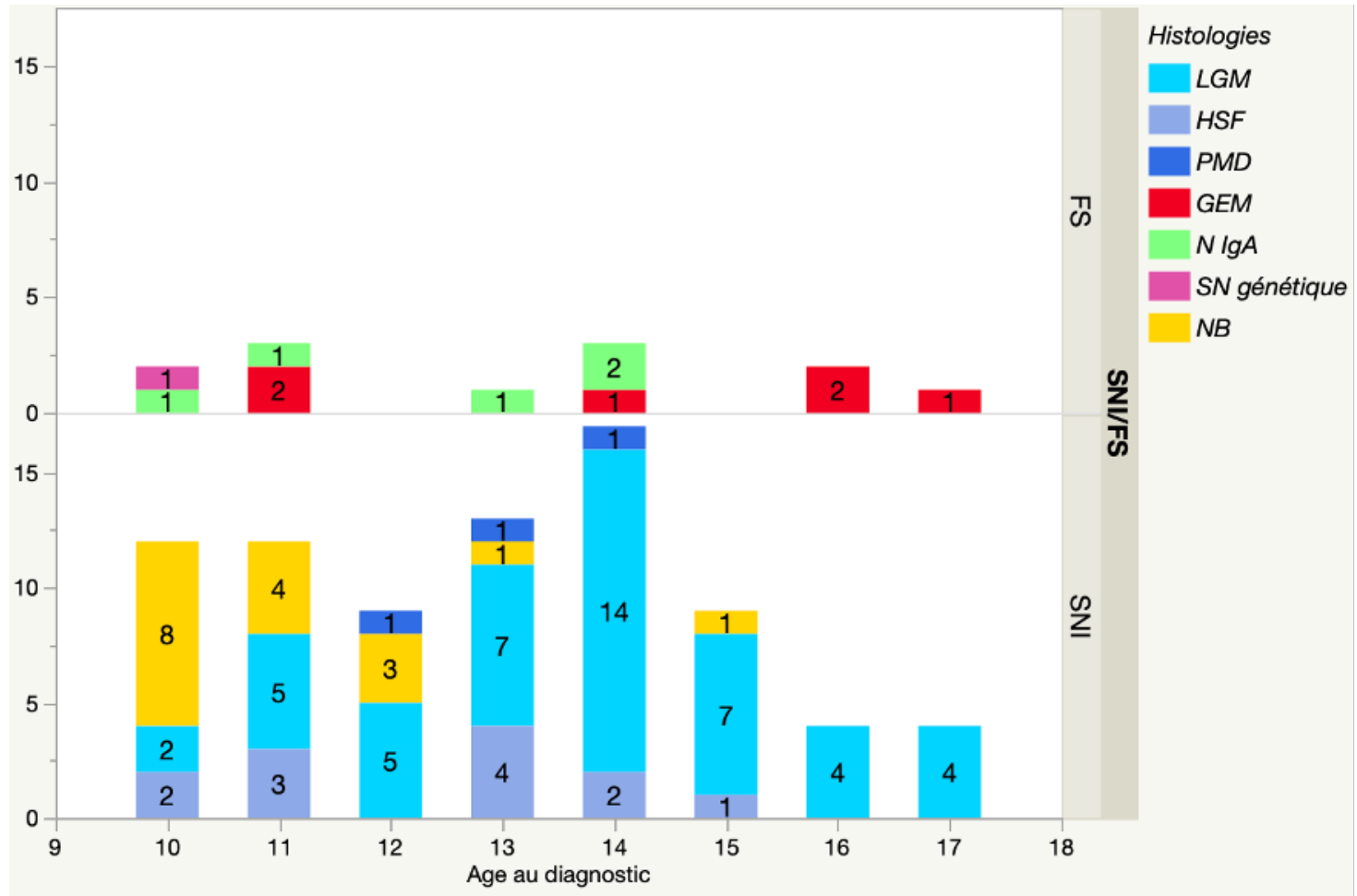

SNI : Syndrome néphrotique idiopathique, FS : Formes secondaires, LGM : Lésions glomérulaires minimes, HSF : Hyalinose segmentaire et focale, PMD : Prolifération mésangiale diffuse, GEM : Glomérulonéphrite extra-membraneuse, NIgA : Néphropathie à IgA, SN : Syndrome néphrotique, NB : Non biopsiés. 
La répartition des étiologies et des lésions histologiques des $\mathrm{SN}$ en fonction des origines ethniques est présentée dans la Figure 5. Nous observons plus de lésion d'HSF chez les patients originaires d'Afrique subsaharienne, en comparaison notamment avec la population caucasienne. Par ailleurs, nous n'observons pas de lésion d'HSF au sein de la population asiatique.

Figure 5. Histogramme en fonction des origines ethniques

A. Répartition des SNI et des FS en fonction des origines ethniques

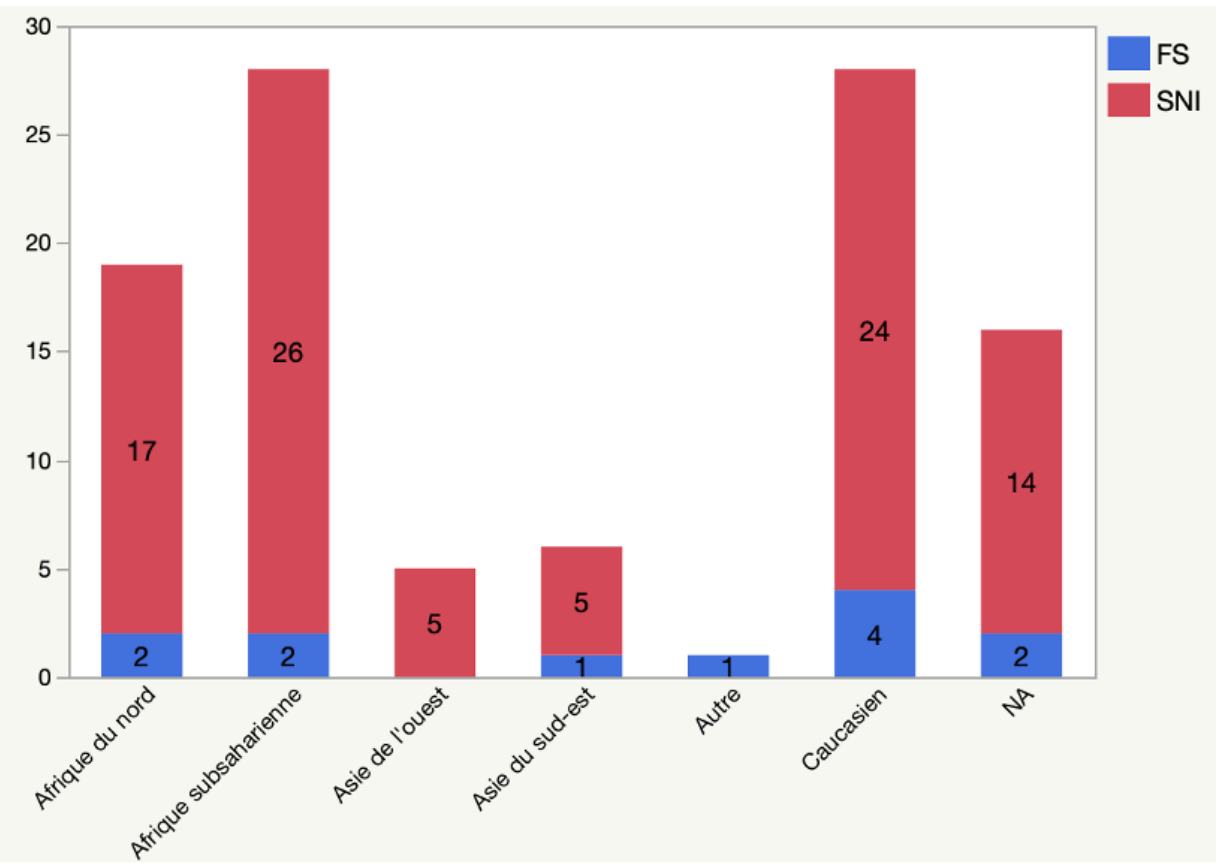

B. Répartition des lésions histologiques en fonction des origines ethniques

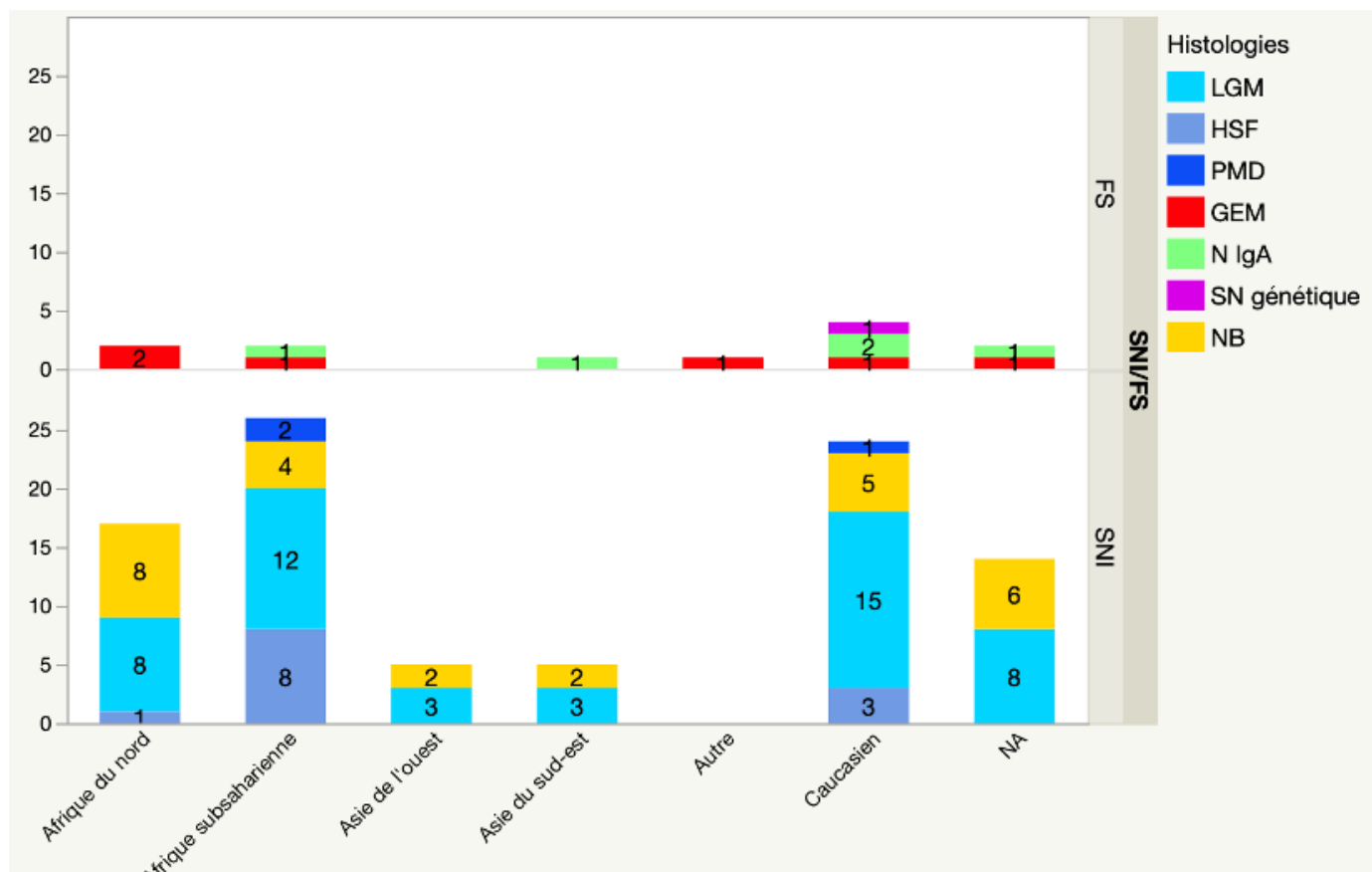

SNI : Syndrome néphrotique idiopathique, FS : Formes secondaires, LGM : Lésions glomérulaires minimes, HSF : Hyalinose segmentaire et focale, PMD : Prolifération mésangiale diffuse, GEM : Glomérulonéphrite extra-membraneuse, NIgA : Néphropathie à IgA, SN : Syndrome néphrotique, NB : Non biopsiés. 


\section{Description des complications des biopsies}

Sur l'ensemble de la population biopsiée, $14,5 \%$ des PBR ont présenté une complication. Il s'agissait exclusivement de complication de type hémorragique : 2 hématuries macroscopiques (2,6\%), 1 épisode de colique néphrétique sur caillot $(1,3 \%), 8$ hématomes péri-rénaux ou sous capsulaires (10,5\%) dont un avec fistule artério-veineuse spontanément résolutive. Il n'a pas été décrit de déglobulisation nécessitant une transfusion ou se compliquant d'un choc hémorragique. Aucune infection du site de ponction n'a été rapportée. Aucun décès en lien avec la réalisation d'une PBR n'a été rapporté.

\section{Recherche d'un éventuel impact d'une PBR différée}

Sur les 12 patients présentant une FS, 6 patients ont eu une PBR au diagnostic : Cinq pour des critères d'atypie (3 néphropathies à IgA qui présentaient des croissants à l'histologie, 1 GEM type 1 et 1 GEM de type 2), 1 pour critère d'âge (GEM type 1) qui a présenté une corticorésistance à 1 mois et pour qui des anticorps anti-PLA2R ont été retrouvés.

Les 6 autres patients ont eu une PBR différée :

Trois ont présenté un critère d'atypie associé à une corticorésistance (1 néphropathie à IgA sans croissant à l'histologie, 1 GEM de type 1 et 1 syndrome génétique avec lésion d'HSF), 1 patient a présenté un critère d'atypie (1 GEM de type 1 avec des FAN positifs) et 2 patients ont présenté une corticorésistance isolée (1 GEM de type 1 avec des FAN positifs et 1 néphropathie à IgA sans croissant à l'histologie). 


\section{Discussion}

Nous rapportons dans cette étude multicentrique et rétrospective, une cohorte de 103 enfants et adolescents, âgés de plus de 10 ans au diagnostic d'un SN d'allure idiopathique, pris en charge dans les services de pédiatrie et néphrologie pédiatrique d'Île-De-France.

Le syndrome néphrotique était idiopathique dans $88 \%$ des cas, et secondaire dans seulement $12 \%$ des cas, sans différence entre les enfants de 10 à 12 ans et les adolescents de 13 à 17 ans. Les deux diagnostics différentiels retrouvés dans cette cohorte de patients sélectionnés sur l'allure idiopathique du SN (absence de signes extrarénaux ou biologiques orientant vers une cause secondaire) étaient la GEM (6\%) et la néphropathie à IgA (5\%).

Chez l'enfant de moins de 10 ans, le SNI est la cause de plus de $90 \%$ des syndromes néphrotiques et est sensible aux corticoïdes dans $90 \%$ des cas, avec une rémission de la protéinurie après un délai médian de 10 jours. ${ }^{18}$ Le traitement de la première poussée repose sur la corticothérapie et le pronostic rénal est excellent, avec néanmoins un risque élevé de rechutes et d'évolution prolongée. ${ }^{19}$ Dans cette population, la biopsie rénale n'a pas d'intérêt diagnostique, puisque le test thérapeutique se suffit à lui-même, ni pronostique puisqu'il n'y a pas de corrélation entre la lésion histologique (LGM et HSF) et l'évolution du syndrome néphrotique. ${ }^{20}$

Chez l'adolescent, des études plus anciennes ont rapporté jusqu'à 50\% de cause secondaire. ${ }^{4}$ Nous avons mis en évidence seulement $11,6 \%$ de formes secondaires. Nous pouvons expliquer cette disparité du fait de l'exclusion initiale dans notre cohorte des sujets présentant une forte suspicion de forme secondaire au diagnostic (signes extra-rénaux cliniques ou biologiques, signes rénaux chroniques évoquant une maladie rénale sous-jacente). Nos résultats ne permettent donc pas d'estimer la fréquence des formes secondaires chez l'adolescent. Nous pouvons cependant remarquer qu'une proportion non négligeable de FS est indiscernable d'un SNI au diagnostic.

Chez l'adulte, le syndrome néphrotique n'est idiopathique que dans $20 \%$ des cas ${ }^{9,10}$ et la première cause de syndrome néphrotique est la GEM. Au cours des 10 dernières années, la découverte de l'antigène PLA2R responsable d'environ 70 à $80 \%$ des GEM primitives de l'adulte ${ }^{13-15}$ a modifié la prise en charge diagnostique et thérapeutique du SN de l'adulte. La biopsie rénale jusque-là systématique devant un syndrome néphrotique de l'adulte, tend à être évitée en cas de sérologie PLA2R positive confirmant le diagnostic de GEM primitive à anticorps anti-PLA2R, mais reste indispensable en cas de $\mathrm{SN}$ avec une sérologie négative. ${ }^{14,15}$ 
Dans la population pédiatrique, la biopsie est réalisée au moment du diagnostic en cas d'atypie, ou secondairement après 4 semaines de corticothérapie orale $+/$ - perfusions de methylprednisolone en cas de corticorésistance. Elle est aussi recommandée au diagnostic sur le critère d'âge $>10$ ou 12 ans. ${ }^{8,21}$

Dans cette cohorte d'enfants de plus de 10 ans au diagnostic, pris en charge dans les services de pédiatrie ou néphrologie pédiatrique d'Île-De-France, nous avons mis en évidence que près de $50 \%$ des patients n'ont pas eu de biopsie rénale au diagnostic et plus de $25 \%$ ne seront jamais biopsiés. De plus, nous avons observé que les patients non biopsiés (Table 3) étaient significativement plus jeunes que les patients biopsiés. Ces constatations reflètent l'attitude actuelle de restreindre ce geste invasif et le questionnement des pédiatres vis-à-vis des recommandations de biopsie sur le seul critère d'âge.

L'organisation des soins en Île-De-France est telle que tout enfant se présentant avec un syndrome œdémateux est référé à un des 35 services de pédiatrie générale ou des 3 services de néphrologie pédiatrique pour le diagnostic et la prise en charge initiale d'un syndrome néphrotique. Après le diagnostic d'un SN d'allure idiopathique, l'indication d'une biopsie rénale conduit à organiser le transfert du patient dans un des 3 services de néphrologie pédiatrique, pour la réalisation du geste. Pour des raisons d'organisation (disponibilité des lits, jours ouvrables, interruption de l'anticoagulation avant le geste), le transfert et la réalisation de la biopsie rénale peuvent être différés de plusieurs jours après l'initiation de la corticothérapie, ce qui parfois permet, en cas de réponse rapide et complète de la protéinurie sous traitement, de confirmer la forme idiopathique du SN et de sursoir à la biopsie rénale. De plus, Moxey-Mims et al ont démontré, au sein d'une population d'adolescents diagnostiqués SNI, que l'espérance de vie était similaire entre ceux ayant bénéficié d'une PBR au diagnostic et ceux ayant reçu une corticothérapie initiale. ${ }^{22}$ Ainsi, une PBR différée d'un mois ne semble pas être associée à une perte de chance pour un patient présentant un SNI.

L'objectif de la biopsie rénale est le diagnostic différentiel d'une cause secondaire du syndrome néphrotique, relevant d'une prise en charge diagnostique et thérapeutique différente de celle du SNI. En ce qui concerne le traitement des principaux diagnostics différentiels du SNI (GEM, glomérulonéphrite à dépôts de C3, néphropathie à IgA ou maladie de Berger), il n'existe pas de consensus national ou international, cependant la $1^{\text {ère }}$ ligne de traitement en cas de SN repose aussi sur les corticoïdes. De plus, les signes histologiques permettant de poser un diagnostic étiologique et un diagnostic de sévérité ne seront pas ou peu altérés par la corticothérapie orale. Même si d'autres marqueurs biologiques (négativation de la sérologie PLA2R, normalisation du complément sont possible après quelques jours ou semaines de corticothérapie), nous assumons que différer la biopsie rénale ne porte pas préjudice au diagnostic et au traitement des FS de SN d'allure idiopathique. 
Dans notre étude, en l'absence de tableau atypique ou de corticorésistance, aucune forme secondaire n'a été décelée. Il s'agissait exclusivement de SNI, et ces patients auraient pu être exemptés de PBR. En revanche, en cas de tableau atypique au diagnostic, un tiers des patients avaient une forme secondaire de SN (Figure 3). Cette fréquence élevée de FS justifie, selon nous, la réalisation d'une PBR d'emblée en cas de tableau atypique, comme chez l'enfant plus jeune. La fréquence des formes secondaires s'élève aussi à plus de $25 \%$ en cas de corticorésistance isolée. A l'inverse, près de $3 / 4$ des patients cortico-résistants (SNI et FS) ne présentaient pas d'atypie au diagnostic. Ainsi, la résistance aux corticoïdes est une indication pertinente de PBR.

La fréquence de l'hématurie microscopique entre les patients corticosensibles et les patients corticorésistants et celle entre les patients présentant un SNI et une FS sont similaires.

L'hématurie microscopique ne semble pas être un critère de PBR ni un facteur prédictif de corticorésistance.

En ce qui concerne l'histologie des SNI, nous avons mis en évidence $76,6 \%$ de LGM et $18,8 \%$ d'HSF. Ces chiffres se rapprochent de ceux retrouvés dans la littérature, renforçant la validité externe de nos résultats. ${ }^{20,21,23}$ Notre étude qui portait sur des adolescents avec un recrutement multi-ethnique met en évidence la LGM comme lésion majoritaire et ne semble pas montrer d'augmentation de lésion d'HSF avec l'âge.

Concernant les origines ethniques, nous avons observé une proportion accrue d'HSF dans la population subsaharienne $(28,6 \%)$ en comparaison à la population caucasienne $(10,7 \%)$. Ces résultats sont cohérents avec ceux de la littérature, dans laquelle plusieurs auteurs ont mis en évidence que les lésions d'HSF étaient la lésion histologique prédominante dans la population d'origine AfroAméricaine. ${ }^{24,25}$ Plusieurs autres études ont également rapporté une proportion prédominante d'HSF dans la population indienne ou pakistanaise. ${ }^{12,26}$ Nos résultats ne permettent pas de constater ces observations, probablement en raison d'un faible recrutement de cette population. Enfin, dans cette cohorte, il n'y a pas de différence de répartition des formes secondaires en fonction de l'ethnie.

Étant donné que l'histologie n'est pas prédictive de la réponse aux corticoïdes et de l'évolution ultérieure, la stratégie thérapeutique de la corticothérapie au cours du premier mois n'est pas modifiée par le résultat de l'histologie précoce. De plus, la seconde ligne de traitement en cas corticorésistance n'est pas non plus conditionnée par le type histologique. ${ }^{19}$

Compte tenu des différences de fréquences des formes secondaires rapportées dans la littérature entre l'enfant et l'adulte, nous nous serions attendus à observer des FS majoritairement après la puberté. Nos résultats ne le mettent pas en évidence (Figure 4). Nous pouvons soit supposer qu'il n'existe pas de répartition préférentielle des FS selon l'âge après 10 ans et notamment après la puberté, soit que le nombre de FS dans notre cohorte était insuffisant pour le mettre en évidence, soit qu'un biais de 
sélection s'est appliqué dans le groupe des patients de 15 ans et plus qui peuvent avoir été pris en charge dans un circuit de néphrologie adulte.

Nous avons observé une différence d'âge de 1 an entre le groupe A et le groupe B, différence statistiquement significative, mais ne semblant pas avoir de pertinence clinique et possiblement expliquée par une plus grande tendance à ne pas réaliser la biopsie systématique chez les enfants de 10 -12 ans (Figure 4).

La biopsie rénale chez les enfants nécessite toujours un transfert dans un centre spécialisé. C'est un geste invasif, réalisé sous anesthésie locale le plus souvent, avec un risque de complication notamment hémorragique. Nammalwar et al. retrouvait 17\% d'hématurie macroscopique, $6 \%$ d'hématomes rénaux et $0,8 \%$ d'infection du site de ponction. ${ }^{16}$ Dans notre cohorte, nous avons retrouvé $14,6 \%$ de complications médicales, exclusivement hémorragiques dont 2 hématuries macroscopiques (2,6\%), 1 épisode de colique néphrétique sur caillot (1,3\%), 8 hématomes (10,5\%). Le caractère rétrospectif de notre étude ne permettait pas d'évaluer l'impact psychologique, social et économique de la biopsie rénale et de ses éventuelles complications.

Considérant que dans notre étude près de la moitié des biopsies réalisées auraient pu être évitées, une limitation des indications des PBR permettrait une réduction du risque de complication mais également d'exposition à des produits anesthésiants, de prolongation d'hospitalisation et de coût. 
Les résultats discutés ci-dessus nous amènent ainsi à proposer dans notre population d'enfants âgés de plus de 10 ans la démarche diagnostique présentée dans la Figure 6, qui correspond aussi à la démarche diagnostique chez les enfants de 1 à 10 ans.

Figure 6. Proposition d'un algorithme de prise en charge diagnostique chez les enfants de plus de 10 ans présentant un syndrome néphrotique

\section{Découverte de syndrome néphrotique entre 10 et 18 ans}

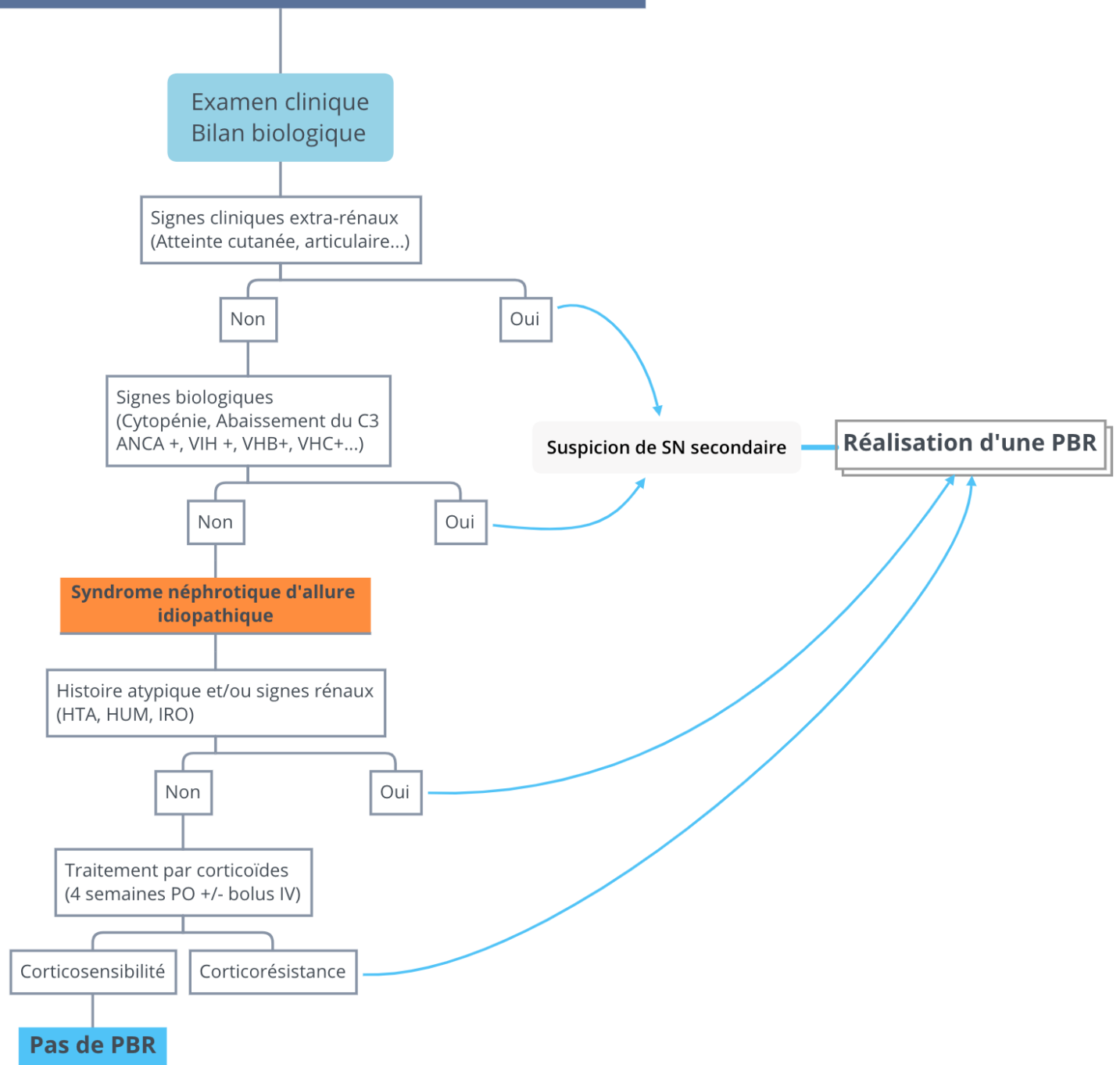

PBR : Ponction biopsie rénale, SN : Syndrome néphrotique, HTA : Hypertension artérielle, HUM : Hématurie macroscopique, IRO : Insuffisance rénale organique, PO : Per os, IV : Intraveineux. 
Notre étude a pour force de se baser sur un recueil de données à la fois histologiques et clinico-biologiques. Elle est l'une des rares permettant de rapporter l'incidence des formes secondaires de SN chez l'adolescent se présentant avec un SN d'allure idiopathique, ce qui permet de rediscuter l'indication de la biopsie rénale et guider le clinicien dans sa prise en charge initiale.

Notre étude présente cependant quelques limites. Il s'agissait d'un recueil rétrospectif, avec certaines données qui restaient manquantes ayant pour conséquence l'exclusion de 7 patients.

Un biais de recrutement est aussi lié à l'âge des patients, en particulier chez les grands adolescents. En effet, avant l'âge de 15 ans, tous les patients avec un SN ont été pris en charge dans un service de pédiatrie et le croisement des différentes bases de données disponibles garantit l'exhaustivité dans ce groupe d'âge. En revanche, certains patients de 15 à 17 ans ont pu être pris en charge exclusivement dans un circuit de néphrologie adulte et n'ont pas été inclus. La stratégie diagnostique et thérapeutique en néphrologie adulte n'étant pas la même, nous n'avons pas cherché à inclure les patients des registres de biopsie rénale des services adultes de la région.

Notre analyse se focalisait sur les $\mathrm{SN}$ d'allure idiopathique, ce qui ne permet pas de conclure à l'incidence globale des formes secondaires de SN chez les plus de 10 ans, puisqu'une grande partie des patients étaient exclus d'emblée de notre étude sur la présence d'arguments anamnestiques, cliniques ou biologiques évoquant une forme secondaire.

L'incidence en Île-De-France du SNI de l'adolescent, l'évaluation de la prise en charge thérapeutique, dont la stratégie d'anticoagulation préventive et l'évolution à long terme des patients SNI basés sur cette cohorte fera l'objet de travaux ultérieurs. 


\section{Conclusion}

Cette étude rétrospective portant sur une cohorte en population de plus de 100 patients avec un SN d'allure idiopathique retrouve une fréquence de seulement $12 \%$ de formes secondaires. Elle met en évidence qu'aucune forme secondaire de SN n'est retrouvée en l'absence de tableau atypique et/ou de corticorésistance et que $40 \%$ des biopsies auraient pu être évitées.

Cette étude nous amène à proposer une nouvelle démarche diagnostique dans notre population, c'est à dire que la PBR chez les enfants de 10 à 18 ans présentant un SN d'allure idiopathique soit limitée aux indications suivantes : histoire atypique, présence d'au moins un signe néphrologique (HTA, hématurie macroscopique, IR organique) ou en cas de corticorésistance, comme chez les enfants plus jeunes. 


\section{Bibliographie}

1. Hinkes BG, Mucha B, Vlangos CN, et al. Nephrotic syndrome in the first year of life: two thirds of cases are caused by mutations in 4 genes (NPHS1, NPHS2, WT1, and LAMB2). Pediatrics 2007;119(4):e907-919.

2. Niaudet P. Syndromes néphrotiques congénitaux et infantiles. Néphrologie Thérapeutique 2005;1(1):63-70.

3. Hogan J, Fila M, Baudouin V, Peuchmaur M, Deschênes G, Niel O. Cytomegalovirus infection can mimic genetic nephrotic syndrome: a case report. BMC Nephrol 2015;16.

4. Nephrotic syndrome in children: prediction of histopathology from clinical and laboratory characteristics at time of diagnosis. A report of the International Study of Kidney Disease in Children. Kidney Int 1978;13(2):159-65.

5. Short versus standard prednisone therapy for initial treatment of idiopathic nephrotic syndrome in children. Arbeitsgemeinschaft für Pädiatrische Nephrologie. Lancet Lond Engl 1988;1(8582):380-3.

6. Eddy AA, Symons JM. Nephrotic syndrome in childhood. Lancet Lond Engl 2003;362(9384):629-39.

7. Noone DG, Iijima K, Parekh R. Idiopathic nephrotic syndrome in children. The Lancet 2018;392(10141):61-74.

8. Protocole National de Diagnostic et de Soins (PNDS) - Syndrome néphrotique idiopathique de l'enfant - 2008. Haute Aut Santé 2016;

9. Sahali D, Audard V, Rémy P, Lang P. Syndromes néphrotiques idiopathiques : physiopathologie et prise en charge thérapeutique spécifique chez l'adulte. Néphrologie Thérapeutique 2012;8(3):180-92.

10. Audard V, Lang $\mathrm{P}$, Sahali D. Pathogénie du syndrome néphrotique à lesions glomérulaires minimes. médecine/sciences 2008;24(10):853-8.

11. Protocole National de Diagnostic et de Soins (PNDS) - Syndrome néphrotique idiopathique de l'adulte - 2008. Haute Aut Santé 2014;

12. Gulati S, Sural S, Sharma RK, Gupta A, Gupta RK. Spectrum of adolescent-onset nephrotic syndrome in Indian children. Pediatr Nephrol 2001;16(12):1045-8.

13. Beck LH, Bonegio RGB, Lambeau G, et al. M-type phospholipase A2 receptor as target antigen in idiopathic membranous nephropathy. N Engl J Med 2009;361(1):11-21.

14. Dahan K. Glomérulonéphrites extramembraneuses. EM-Consulte - Néphrologie 2020;32(2):1-8.

15. Bobart SA, Fervenza FC. Kidney Biopsy Is Required for Nephrotic Syndrome with PLA2R+ and Normal Kidney Function: The Con View. Kidney360 2020;1(9):890-3. 
16. Nammalwar BR, Vijayakumar M, Prahlad N. Experience of renal biopsy in children with nephrotic syndrome. Pediatr Nephrol Berl Ger 2006;21(2):286-8.

17. Dossier C, Lapidus N, Bayer F, et al. Epidemiology of idiopathic nephrotic syndrome in children: endemic or epidemic? Pediatr Nephrol Berl Ger 2016;31(12):2299-308.

18. Dossier C, Delbet J-D, Boyer O, et al. Five-year outcome of children with idiopathic nephrotic syndrome: the NEPHROVIR population-based cohort study. Pediatr Nephrol 2019;34(4):671-8.

19. Trautmann A, Vivarelli M, Samuel S, et al. IPNA clinical practice recommendations for the diagnosis and management of children with steroid-resistant nephrotic syndrome. Pediatr Nephrol Berl Ger 2020;35(8):1529-61.

20. Narla D, Swiatecka-Urban A. Therapeutic Response to Corticosteroids Remains a Valid Approach to Initial Management of Children With Idiopathic Nephrotic Syndrome. Front Pediatr 2020;8:533.

21. Gipson DS, Massengill SF, Yao L, et al. Management of childhood onset nephrotic syndrome. Pediatrics 2009;124(2):747-57.

22. Moxey-Mims MM, Stapleton FB, Feld LG. Applying decision analysis to management of adolescent idiopathic nephrotic syndrome. Pediatr Nephrol Berl Ger 1994;8(6):660-4.

23. Ayach G, El-Filali H, Saidi S, et al. Histopathological Study of Pure Primary Nephrotic Syndrome in Adolescents and Young Moroccan Adults. Arab J Nephrol Transplant 2011;4(3):137-40.

24. Baqi N, Singh A, Balachandra S, et al. The paucity of minimal change disease in adolescents with primary nephrotic syndrome. Pediatr Nephrol Berl Ger 1998;12(2):105-7.

25. Bonilla-Felix M, Parra C, Dajani T, et al. Changing patterns in the histopathology of idiopathic nephrotic syndrome in children. Kidney Int 1999;55(5):1885-90.

26. Mubarak M, Kazi JI, Lanewala A, Hashmi S, Akhter F. Pathology of idiopathic nephrotic syndrome in children: are the adolescents different from young children? Nephrol Dial Transplant 2012;27(2):722-6. 


\section{SERMENT D'HIPPOCRATE}

Au moment d'être admis(e) à exercer la médecine, je promets et je jure d'être fidèle aux lois de l'honneur et de la probité.

Mon premier souci sera de rétablir, de préserver ou de promouvoir la santé dans tous ses éléments, physiques et mentaux, individuels et sociaux.

Je respecterai toutes les personnes, leur autonomie et leur volonté, sans aucune discrimination selon leur état ou leurs convictions. J'interviendrai pour les protéger si elles sont affaiblies, vulnérables ou menacées dans leur intégrité ou leur dignité. Même sous la contrainte, je ne ferai pas usage de mes connaissances contre les lois de l'humanité.

J'informerai les patients des décisions envisagées, de leurs raisons et de leurs conséquences. Je ne tromperai jamais leur confiance et n'exploiterai pas le pouvoir hérité des circonstances pour forcer les consciences.

Je donnerai mes soins à l'indigent et à quiconque me le demandera. Je ne me laisserai pas influencer par la soif du gain ou la recherche de la gloire.

Admis(e) dans l'intimité des personnes, je tairai les secrets qui me seront confiés. Reçu(e) à l'intérieur des maisons, je respecterai les secrets des foyers et ma conduite ne servira pas à corrompre les mœurs.

Je ferai tout pour soulager les souffrances. Je ne prolongerai pas abusivement les agonies. Je ne provoquerai jamais la mort délibérément.

Je préserverai l'indépendance nécessaire à l'accomplissement de ma mission. Je n'entreprendrai rien qui dépasse mes compétences. Je les entretiendrai et les perfectionnerai pour assurer au mieux les services qui me seront demandés.

J'apporterai mon aide à mes confrères ainsi qu'à leurs familles dans l'adversité.

Que les hommes et mes confrères m'accordent leur estime si je suis fidèle à mes promesses ; que je sois déshonoré(e) et méprisé(e) si j'y manque. » 
SMITH (Anna) - Syndrome néphrotique d'allure idiopathique après l'âge de 10 ans :

Intérêt de la biopsie rénale systématique au diagnostic

43 pages, 6 figures, 4 tables Th. : Méd. : Brest 2021

\section{RESUME :}

La biopsie rénale est recommandée au diagnostic pour les enfants présentant un syndrome néphrotique (SN) après l'âge de 10 ans car, contrairement à l'enfant plus jeune, la fréquence des formes secondaires du SN est plus élevée. Cette démarche est de plus en plus remise en question.

L'objectif principal était d'étudier l'intérêt de la biopsie rénale systématique dans la stratégie diagnostique chez les enfants de plus de 10 ans présentant un SN.

Nous avons conduit une étude rétrospective, multicentrique, incluant des enfants de 10 à 18 ans de la région île-De-France, présentant un SN entre décembre 2007 et juin 2020.

103 patients inclus ont pu être analysés. Une biopsie rénale a été réalisée pour 76 patients dont 43 présentaient au moins une indication de biopsie autre que l'âge (tableau atypique et/ou corticorésistance). Les résultats histologiques retrouvaient : 49 LGM, 13 HSF, 3 PMD, 6 GEM, 5 néphropathies à IgA.

Aucun SN de forme secondaire n'a été retrouvé chez les 33 patients biopsiés pour critère d'âge seul.

Cette étude rétrospective de plus 100 patients avec un SN d'allure idiopathique retrouve une fréquence de seulement $12 \%$ de formes secondaires. Elle met en évidence qu'aucune forme secondaire de SN n'est retrouvée en l'absence de tableau atypique et/ou de corticorésistance et que $40 \%$ des biopsies auraient pu être évitées.

Nous proposons de limiter la réalisation d'une biopsie rénale chez les enfants de 10 à 18 ans aux indications suivantes : histoire atypique, présence d'au moins un signe néphrologique (hypertension artérielle, hématurie macroscopique, insuffisance rénale organique) ou en cas de corticorésistance.

\section{MOTS CLES :}

Syndrome néphrotique ; adolescents ; ponction biopsie rénale ; stratégie diagnostique

JURY :

Président : M. le Professeur DE PARSCAU DU PLESSIX Loïc

Membres : Mme le Docteur DOSSIER Claire (directrice de thèse)

Mme le Docteur JAY Nadine

Mme le Docteur ROPARS Juliette

M. le Professeur LE MEUR Yannick

\section{DATE DE SOUTENANCE :}

01 juin 2021 